\title{
A Case of Uncontrolled Still's Disease \& Macrophage Activating Syndrome
}

\author{
Ann Eapen, MD ${ }^{1}$, Susan O'Brien, MD ${ }^{1}$
}

Chao Family Comprehensive Cancer Center ${ }^{1}$, Division of Hematology/Oncology University of California, Irvine

\section{BACKGROUND:}

- Macrophage Activating Syndrome (MAS) is a rare and often fatal condition that is a subset of Hemophagocytic Lymphohistiocytosis (HLH). MAS is a form of secondary HLH specifically associated with rheumatologic conditions, such as Still's Disease ${ }^{1}$

- Characterized by Uncontrolled activation of Macrophages and T-lymphocytes with marked Increase of cytokines and decreased NK cell function ${ }^{1}$

- Adult Onset Still's Disease (AOSD) is a rare rheumatologic condition associated with high fevers, joint pain and salmon-colored rash usually treated with steroids, NSAIDs, and DMARDs

- In this unique presentation, a young patient had a delayed diagnosis of AOSD, refractory to standard treatment, eventually resulting in MAS, which led to his untimely demise. Given the poor prognosis of MAS, there is a clear need for improved management and diagnosis of this condition

\section{CASE DESCRIPTION:}

- $\mathrm{Mr} . \mathrm{MZ}$ is a 30 y/o male construction worker with uncontrolled joint pain, fevers, fatigue for a year, with delayed diagnosis and mismanagement of AOSD, who presented to an OSH in April 2018 with fevers, cough, altered mental status. Patient was treated for weeks with antibiotics and low dose steroids at OSH for presumed pneumonia but with continued deterioration, eventually resulting in hypoxic respiratory failure and intubation

- Patient then transferred to $\mathrm{UCl} 3$ weeks after initial presentation and noted to have fevers, cytopenias, acute renal failure, transaminitis, hyper-ferritinemia and notable hepatomegaly on exam

- Suspicion for MAS high: Ferritin >15,000. Fasting TG: 414 AST 2183 ALT 774, Hgb 6.1, creatinine 2.9 IL-2 receptor $9340 .>10.00093 \%$ specific $^{1}$ ESR decline from 50 to 5

\section{- H-score 99.8\%; 95\% without bone marrow biopsy}

- H-SCORE Calculation includes: 1) Fever 2) Splenomegaly 3) Hepatomegaly 4) (Cytopenias 5) Ferritin 6) Triglyceride 7) Fibrinogen 8) AST/ALT 9) Bone Marrow Biopsy

- Treated immediately with high dose steroids with a confirmatory bone marrow biopsy within 12 hours. Unfortunately, patient had cardiac arrest and was placed on hypothermia protocol and treated with MAS management, but ultimately passed away within 36 hours of arrival

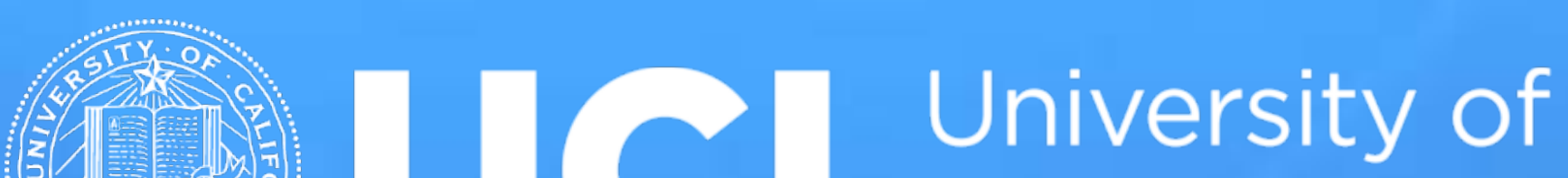
UCl

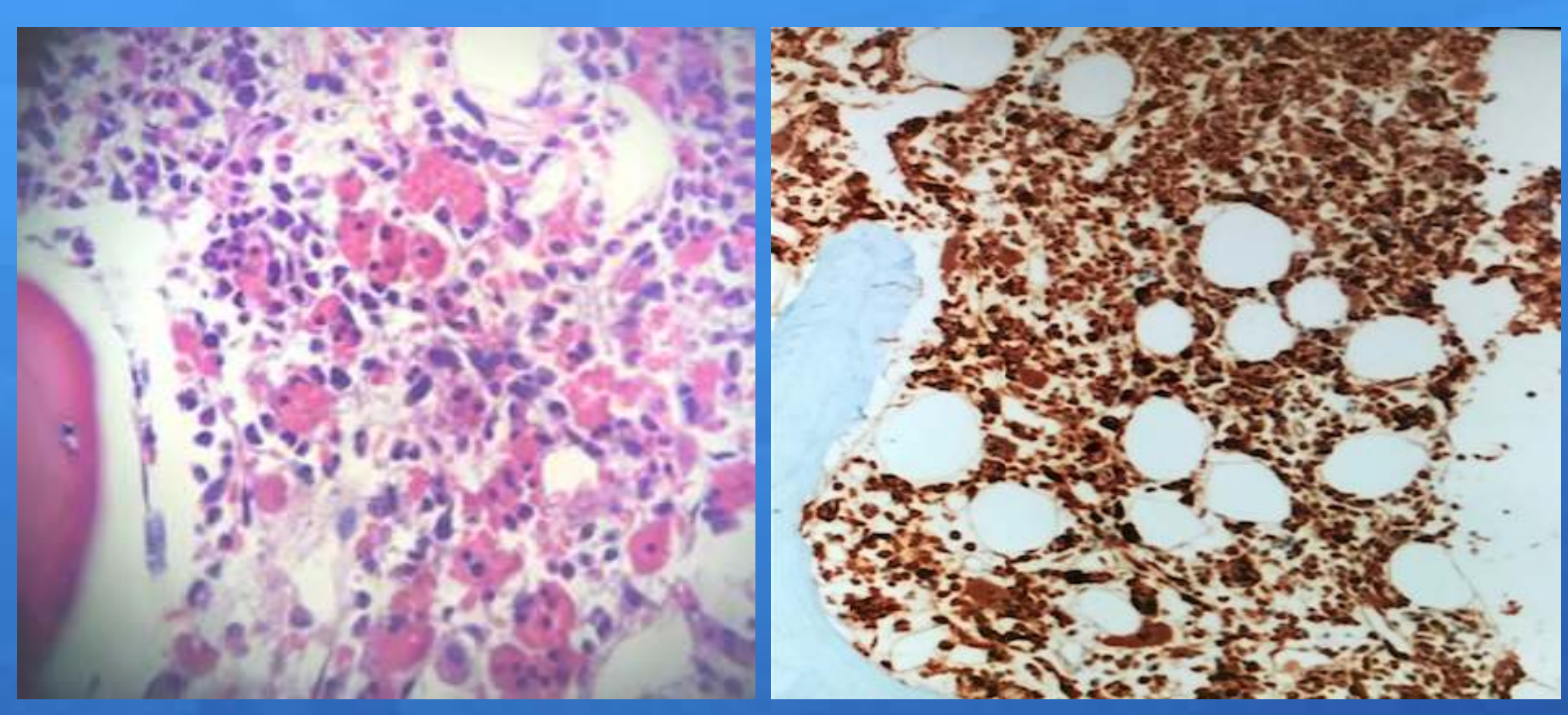

1) Bone Marrow Biopsy findings of patient MZ classic for HLH with macrophages engulfing red blood cells 2) CD68 confirmatory staining for macrophages

\section{DISCUSSION:}

- This presentation delineates a rare case of refractory AOSD resulting in MAS which invites a discussion of key findings and management that would be helpful to decrease future morbidity and mortality for this subset of patients

- MAS has a poor prognosis given its aggressive nature, with a high proliferation and high incidence of involvement in the bone marrow displaying the need for early diagnosis and management

- If AOSD patients progress through standard treatment, biologic agents such as Anakinra ${ }^{3 \prime}$ an IL-1 receptor antagonist and combination therapy with Cyclosporine should be rapidly instituted to prevent MAS3

- Literature review reveals that Tocilizumab after methylprednisolone pulse therapy has been an effective agent ${ }^{2}$ in inducing clinical remission of AOSD-related MAS

- Retrospective analysis performed by Ruscitt et. al illustrates that hyper-ferritinemia was independently associated with an increased probability of MAS3, while Mayo Clinic review by Pham et. al reveal that hyper-ferritinemia in adult patients is more likely to be associated with malignancy, and infection in contrast to the pediatric population 5

- Multicenter studies have shown the H-score to be less restrictive than HLH-2004 criteria. H-score evaluation in adults should be performed on initial presentation to improve accuracy. In children, the $\mathrm{H}$-score has better sensitivity than the HLH-2004 criteria, but both have adequate specificity ${ }^{4}$, and could preclude the need to wait for bone marrow biopsy results, therefore expediting treatment

REFERENCES.
1. Schram, et al. How I treat hemophagocytic lymphohistiocytosis in the adult patient Blood 2015 Volume 125, 2. Watanabe et. Al, Successful Tocilizumab Therapy for Macrophage Activating Syndrome 125, 2. Watanabe et. Al, Successful Tocilizumab Therapy for Macrophage Activating Syndrome
Associated with Adult-Onset Still's Disease, Case Rep Med, 2016 3. Ruscitti et al, Macrophage Activating Syndrome in Still's Disease, Clin Rheum 20 4. Mahadeb et al, Performances of the H-score for Diagnosis of HLH in Adult and Pediatric Patients. Am J Clin Path June 2016 5. Pham et al. Analysis of Serum Ferritin Levels as a Diagnostic Criteria for HLH hospitalized patients Blood 2015 126:1014 